\title{
The precision of velocity discrimination across spatial frequency
}

\author{
YUE CHEN \\ Harvard University, Cambridge, Massachusetts \\ and \\ HAROLD E. BEDELL and LAURA J. FRISHMAN \\ University of Houston, Houston, Texas
}

\begin{abstract}
The precision of velocity coding for moving stimuli of different spatial frequencies was assessed by measuring velocity discrimination thresholds for a 1-c/deg grating paired with a grating whose spatial frequency ranged from 0.25 to $4 \mathrm{c} / \mathrm{deg}$ and for grating pairs of the same spatial frequency $(0.25,1$, and $4 \mathrm{c} / \mathrm{deg}$ ). The gratings always moved upward, with velocities ranging from 0.5 to $16 \mathrm{deg} / \mathrm{sec}$. Velocity discrimination was as precise for stimuli that varied in spatial frequency by \pm 2 octaves $(0.25 \mathrm{vs} .1 \mathrm{c} / \mathrm{deg}$ and $4 \mathrm{vs} .1 \mathrm{c} / \mathrm{deg}$ ) as for stimuli of the same spatial frequency, for specific ranges of velocity that depended on the spatial and, therefore, the temporal frequencies of the stimuli. Compared with a 1-c/deg grating, the perceived velocity of 4-c/deg gratings was about 1.3 times faster and that of 0.25 -c/deg gratings was about 1.3 times slower. Although these perceived velocity biases imply variation of velocitysignal processing among spatial frequency channels, the discrimination results indicate that the motionsensing system can compare signals across different spatial frequency channels to make fine velocity discrimination within appropriate temporal frequency limits.
\end{abstract}

The coding of velocity by the visual system is an important aspect of motion processing. In physical terms, the velocity of a narrow-band stimulus is related to its spatial frequency via the ratio of temporal frequency (TF) to spatial frequency (SF) - that is, velocity $=$ TF/SF. Physiological and psychophysical studies indicate that the processing of spatial and temporal frequency information at early stages of the visual system is mediated by multiple mechanisms, each of them tuned to a limited range of spatial and temporal frequencies (see, e.g., Blakemore \& Campbell, 1969; DeValois, Albrecht, \& Thorell, 1982; Movshon, Thompson, \& Tolhurst, 1978; Wilson, McFarlane, \& Philip, 1984). However, moving targets are often spatially broadband, making the extent to which velocity coding is invariant across spatial frequency an important issue.

To investigate velocity coding across spatial frequency in human observers, McKee, Silverman, and Nakayama (1986) examined the effect of random changes in spatial frequency, between $0.5-1.5 \mathrm{c} / \mathrm{deg}$, on velocity discrimination for stimuli moving at one velocity, $5 \mathrm{deg} / \mathrm{sec}$. They

This work was supported in part by NIH Training Grant EY07024, Research Grants EY06671 and EY05068, and a VRSG award from UHCO. We thank Dennis M. Levi and Haluk Ögmen for helpful discussions concerning this study, a portion of which was presented at the 1995 ARVO meeting. We also thank the anonymous referees for their useful suggestions and thoughtful comments. Correspondence concerning this article should be addressed to H. E. Bedell, College of Optometry, University of Houston, Houston, TX 77024-6052 (e-mail: hbedell@, uh.edu). found that discrimination remained quite precise for the range of spatial (and, hence, temporal) frequencies that they used. As they pointed out, this result supports the notion that a velocity signal per se, rather than simply the grating's temporal frequency, is involved in velocity discrimination. Smith and Edgar (1991) used a similar spatial frequency randomization paradigm to investigate the range over which this velocity coding occurs. They found that discrimination was degraded at velocities greater than about $5 \mathrm{deg} / \mathrm{sec}$ when the stimulus spatial frequencies were randomized even over a small range $(0.875-1.125 \mathrm{c} / \mathrm{deg})$. A similar high-velocity limit in a previous study led Smith (1987) to suggest that velocity coding may occur only at low and intermediate velocities, whereas, at high velocities, an explicit temporal frequency code mediates discrimination.

Velocity coding that is relatively stable over spatial frequency may develop at cortical stages that are specialized for motion processing, upstream from those where tuning is restricted to a limited range of spatial and temporal frequencies. Two-stage models based on a segregation of this kind have been proposed for velocity processing (Grzywacz \& Yuille, 1990; Heeger, 1987). At the first stage, temporal and spatial signals generate velocity information from the relationship, velocity $=\mathrm{TF} / \mathrm{SF}$. At the second stage, information is pooled from different spatial frequency channels in the first stage to produce speed coding that is spatial frequency invariant. Some neural units of the middle temporal (MT) area of the macaque monkey (Newsome, Gizzi, \& Movshon, 1983) and at least some V1 neurons that project to MT (Movshon \& Newsome, 
1996) have broader spatial bandwidths than the remaining motion-sensitive units in V1. Further, MT units are sharply tuned to the velocity of a moving bar (a broadband stimulus); overall, the distribution of preferred velocities in MT is unimodal, peaking near $32 \mathrm{deg} / \mathrm{sec}$ (Maunsell \& van Essen, 1983). Orban, de Wolf, and Maes (1984) showed that human observers are able to perform precise velocity discrimination up to $30-60 \mathrm{deg} / \mathrm{sec}$ for a moving bar, much higher velocities than those for which Smith (1987; Smith \& Edgar, 1991) found evidence for velocity coding. However, because the multiple spatial components of a moving broadband stimulus also correspond to a range of temporal frequencies, it is not possible, using bar stimuli, to distinguish between discrimination based on a velocity code and discrimination based on a temporal frequency code.

In order to clarify the limits of the range over which velocity coding mediates precise velocity discrimination, we assessed velocity discrimination when the spatial frequency difference between two comparison gratings was varied for base velocities between 0.5 and $16 \mathrm{deg} / \mathrm{sec}$ and a wider range of spatial frequencies ( \pm 2 octaves) than those used in previous studies (McKee et al., 1986; Smith \& Edgar, 1991). Gratings that differ in spatial frequency by 2 octaves presumably stimulate different spatial frequency mechanisms in early visual processing. We found that velocity discrimination was as precise for stimuli that varied in spatial frequency by \pm 2 octaves $(0.25$ vs. $1 \mathrm{c} / \mathrm{deg}$ and $4 \mathrm{vs} .1 \mathrm{c} / \mathrm{deg}$ ) as for stimuli of the same spatial frequency, for specific ranges of velocity. The deterioration of precise velocity discrimination at high velocities when high spatial frequencies were used and at low velocities when low spatial frequencies were used is consistent with a temporal frequency limitation on velocity coding.

\section{METHOD}

\section{Stimulus}

The stimulus was a patch of moving sinusoidal grating inside of a 6-deg sharp circular aperture, generated on a Mitsubishi CRT by a Cambridge VSG 2.2 board based in a PC clone 386 computer. The display was fixed at $1.7 \mathrm{~m}$ away from the observer, which produced a total angular size of about $12 \times 10 \mathrm{deg}$. The mean luminance of the grating and surrounding field was $50 \mathrm{~cd} / \mathrm{m}^{2}$ and the frame rate was $70 \mathrm{~Hz}$. On each trial, two horizontal gratings were presented moving upward, one after another. ${ }^{1}$ One grating, referred to as the reference, had a spatial frequency of $1 \mathrm{c} / \mathrm{deg}$ (except when indicated otherwise), and the other grating, referred to as the comparison, had one of five spatial frequencies $(0.25,0.5,1,2$, or $4 \mathrm{c} / \mathrm{deg})$ in different experimental runs. Because of the circular aperture, the spatial bandwidths of the grating stimuli ranged from 0.9 octaves (full width at half height) at $0.25 \mathrm{c} / \mathrm{deg}$ to 0.05 octaves at $4 \mathrm{c} / \mathrm{deg}$. The presentation order of the reference and the comparison gratings was randomized from trial to trial. The overall range of velocities tested was generally from 0.5 to $16 \mathrm{deg} / \mathrm{sec}$, except for trials using a $0.25-\mathrm{c} / \mathrm{deg}$ grating, for which the range was extended to $32 \mathrm{deg} / \mathrm{sec}$. Temporally, each stimulus was presented within a $200 \mathrm{msec}$ raised sinusoidal window. Tracking eye movements were minimized by the relatively brief duration of the stimuli and by a bright cross at the center of the display, which the observers were instructed to fixate.
The time interval between the two presentations was 1 sec. To minimize the influence of temporal cues on velocity discrimination, the spatial frequencies and, therefore, the temporal frequencies of both gratings were jittered by $\pm 10 \%$ from presentation to presentation. To minimize contrast cues for perceived velocity, each grating's contrast was also jittered by $\pm 10 \%$ of the nominal value ( $40 \%$ contrast).

\section{Procedure}

A two-alternative temporal forced choice procedure was used to determine the velocity discrimination threshold. This procedure was combined with a double staircase, one to determine an incremental velocity and the other to determine a decremental velocity Defined as just distinguishably faster or slower than the reference velocity, the incremental and decremental velocities were used to compute relative velocity biases when velocity comparisons were made between different spatial frequencies, and they ensured that these biases did not affect velocity discrimination. The velocity discrimination threshold and the relative velocity bias were calculated, separately, by taking half of (1) the difference and (2) the sum of the incremental and decremental velocities, respectively:

$$
\begin{gathered}
\Delta V=\left(V_{\mathrm{i}}-V_{\mathrm{d}}\right) / 2 \\
V_{\mathrm{B}}=\left(V_{\mathrm{i}}+V_{\mathrm{d}}\right) / 2
\end{gathered}
$$

Here, $V_{\mathrm{i}}$ and $V_{\mathrm{d}}$ designate the incremental and decremental velocities, $\Delta V$ represents the discrimination threshold, and $V_{\mathrm{B}}$ represents the velocity of the comparison perceived to be equal to the velocity of the reference. Because of the velocity biases when the reference and comparison gratings had different spatial frequencies (Ferrera \& Wilson, 1991; McKee et al., 1986; Smith \& Edgar, 1990; also see below), $V_{\mathrm{i}}$ and $V_{\mathrm{d}}$ could be either greater or smaller than the velocity of the reference grating.

To measure $V_{i}, 50 \%$ of the reference velocity was first added to the comparison stimulus as an initial increment. The velocity of the comparison was decreased or increased by $5 \%$ when the observer correctly or incorrectly indicated which of the two stimulus presentations in one trial contained the greater velocity. The decreasing step size for the velocity of the comparison velocity was reduced to $2.5 \%$ following the first staircase reversal (the increasing step size was kept at $5 \%$ ). This decision rule tracks the threshold corresponding to about $71 \%$ correct. Thirty trials for the incremental measurement (along with 30 trials for the decremental measurement; see below), terminated one experimental run. The mean of the lower reversals plus half of the last decreasing staircase step size produced one estimate of $V_{i}$, unless the number of reversals was less than three, in which case the measurement was repeated.

Except for starting with a $50 \%$ decrement of the reference velocity, the procedure for determining $V_{\mathrm{d}}$ was similar to that for the incremental velocity. Initially, the velocity of the comparison was increased or decreased by $5 \%$, according to the correctness of observer's velocity judgment. After the first reversal, the increasing step size of the comparison velocity was changed to $2.5 \%$, while the decreasing step size was kept at $5 \%$.

The measurement of $V_{\mathrm{i}}$ and $V_{\mathrm{d}}$ was conducted concurrently by randomly interleaving incremental and decremental trials within one experimental run (i.e., 60 trials in total). This arrangement made the velocities of the comparison stimulus, which had a specific spatial frequency, either lower or higher than those of the reference on successive trials; therefore, observers could not anticipate velocity from the associated spatial frequencies. For each observer, four estimates of $V_{\mathrm{i}}$ and $V_{\mathrm{d}}$ were collected in separate runs. On the basis of the four pairs of $V_{\mathrm{i}}$ and $V_{\mathrm{d}}$, four estimates for the discrimination threshold $(\Delta V)$ and relative velocity bias $\left(V_{\mathrm{B}}\right)$ were derived, and their geometric means were taken as the discrimination threshold and relative velocity bias for each combination of spatial frequency difference and velocity. The discrimination threshold was expressed as a Weber fraction: $\Delta V / V_{\mathrm{B}}$. 

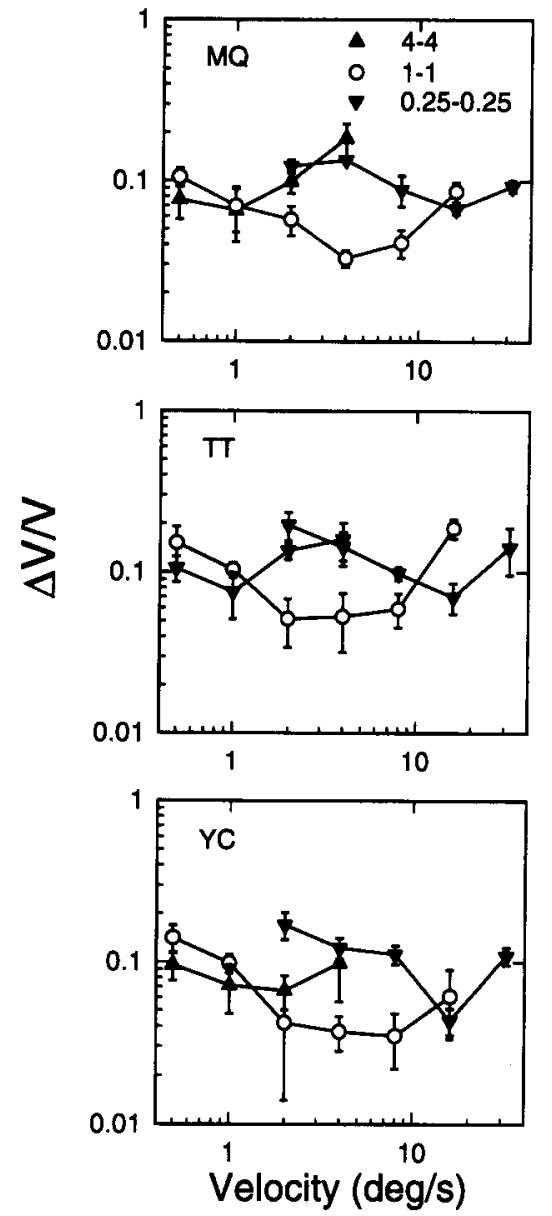

Figure 1. Velocity discrimination thresholds $(\Delta V / V)$ for comparison stimuli of the same spatial frequency. In all three panels (top, M.Q.; middle, T.T.; and bottom, Y.C.), the unfilled circles and the filled triangles and inverted triangles represent data obtained with gratings of 1,4 , and $0.25 \mathrm{c} / \mathrm{deg}$, respectively. The error bars show $\pm 1 S E M$.

\section{Observers}

Three adults with normal or corrected-to-normal vision served as observers. Two of them (M.Q. and T.T.) had no knowledge of the experimental hypothesis. T.T. also had little previous experience in psychophysical experiments. The observers were instructed to indicate, for both incremental and decremental trials, which of the two gratings moved faster. They were advised specifically to overlook any apparent spatial and temporal frequency differences between the two gratings within each trial and to make judgments solely on the basis of velocity. Substantial practice, without feedback, was provided before data collection began. The experiment was conducted binocularly.

\section{RESULTS}

\section{Velocity Discrimination Thresholds}

The empirical goal of this study was to evaluate the effect of a spatial frequency difference on the velocity discrimination threshold for pairs of gratings. To establish benchmarks against which effects of spatial fre- quency differences could be evaluated, velocity discrimination thresholds were measured initially with grating pairs of the same spatial frequency. Figure 1 shows discrimination thresholds, expressed as $\Delta V / V$, for pairs of gratings of the same spatial frequency (designated as 1-1, $4-4$, and $0.25-0.25$, for $1 \mathrm{vs} .1 \mathrm{c} / \mathrm{deg}, 4 \mathrm{vs} .4 \mathrm{c} / \mathrm{deg}$, and 0.25 vs. $0.25 \mathrm{c} / \mathrm{deg}$ gratings, respectively). In general, in the 1-1 condition, $\Delta V / V$ for all 3 observers reaches a minimum of about $0.04-0.05$ at an intermediate range of velocities ( $2-8 \mathrm{deg} / \mathrm{sec})$ and increases at lower and higher velocities. It is obvious that the velocity range for optimal discrimination is spatial frequency dependent. For the lowest spatial frequency pair $(0.25-0.25)$, discrimination is better at high velocities, whereas, for the highest spatial frequency pair (4-4), discrimination is better at low velocities. The overall U-shaped functions are in agreement with the results of previous studies (see, e.g., McKee et al., 1986). No data were obtained at high velocities under the 4-4 condition or at low velocities under the $0.25-0.25$ condition, because velocity discrimination for these stimuli was severely degraded.

The velocity discrimination thresholds of the same 3 observers for five pairs of gratings with different spatial frequency combinations are shown in Figure 2. Designated as $1-0.25,1-0.5,1-1,1-2$, and $1-4$, the five conditions correspond to a reference grating of $1 \mathrm{c} / \mathrm{deg}$ versus a comparison grating of $0.25,0.5,1,2$, or $4 \mathrm{c} / \mathrm{deg}$. The main results are that discrimination for the $1-2$ and 1-4 conditions is most precise at low velocities and deteriorates for velocities greater than about $4 \mathrm{deg} / \mathrm{sec}$, similar to the results for the 4-4 condition (cf. Figure 1). Conversely, discrimination for the $1-0.25$ and $1-0.5$ conditions is most precise at high velocities and deteriorates for velocities less than 4-8 deg/sec, more similar to the results for the $0.25-0.25$ condition, for which precision was best at $16 \mathrm{deg} / \mathrm{sec}$ (cf. Figure 1).

The interaction between spatial frequency and velocity on velocity discrimination is further illustrated in Figure 3, which shows ratios of the pooled discrimination thresholds for all 3 observers obtained in the $1-n$ condition ( $n$ denotes a number other than 1 ) and those obtained in the $1-1$ condition, expressed as $\log [(\Delta V / V$ for $1-n) /(\Delta V / V$ for 1-1)]. The horizontal dashed line in each panel in the figure corresponds to a ratio of 1, representing equal discrimination thresholds for the compared conditions. The data points above the horizontal line mean that thresholds are higher for the unequal spatial frequency conditions than for the 1-1 condition. The points below the horizontal line mean the opposite. Figure 3a shows plots of the discrimination threshold ratios for the comparison gratings whose spatial frequencies were higher than that of the reference grating $(1 \mathrm{c} / \mathrm{deg})$. In general, the threshold ratios increase with velocity, indicating degraded velocity discrimination for the 1-4 and 1-2 conditions at the higher velocities (e.g., $4-8 \mathrm{deg} / \mathrm{sec}$ ). In contrast, a decrease of threshold ratios with velocity is shown in Figure 3b, which plots the ratios for the comparison gratings whose spatial frequencies are lower than 

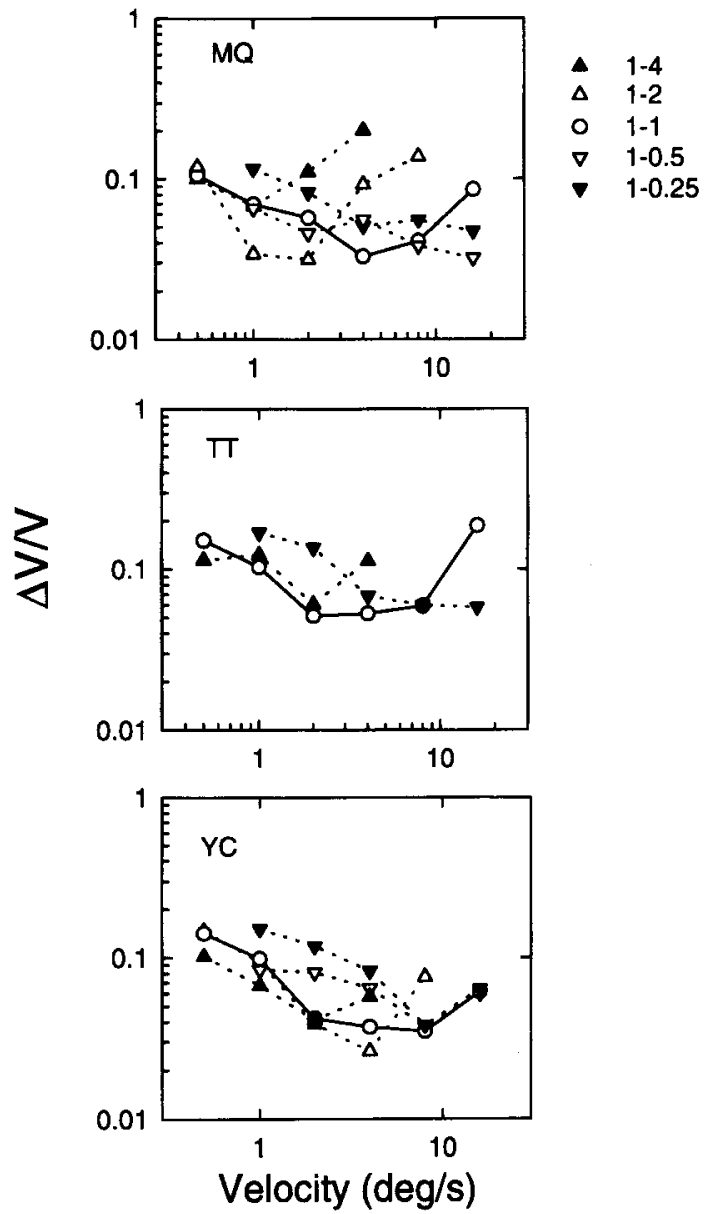

Figure 2. Velocity discrimination thresholds $(\Delta V / V)$ for stimulus pairs of different spatial frequencies. Different curves (five for M.Q., top, and Y.C., bottom, and three for T.T., middle) in each panel correspond to the data obtained under the following spatial frequency combination: $1-4,1-2,1-1,1-0.5$, and $1-0.25$. For clarity, error bars are not presented; the average values of all measured standard errors are 0.014 for M.Q., 0.022 for T.T., and 0.019 for Y.C.

the reference grating. In fact, Figure $3 b$ indicates that the velocity discrimination for the $1-0.25$ and $1-0.5$ conditions is most precise at high velocities (e.g., $16 \mathrm{deg} / \mathrm{sec}$ ) and even better than that for the 1-1 condition for 2 of the 3 observers.

The finding that precise velocity discrimination is maintained for stimuli of different spatial frequencies was tested further for two observers (M.Q. and Y.C.), using our largest spatial frequency difference, a reference of $0.25 \mathrm{c} / \mathrm{deg}$, and a comparison of $4 \mathrm{c} / \mathrm{deg}$ (designated as $0.25-4$ ). The measurements were taken only at velocities of 2 and $4 \mathrm{deg} / \mathrm{sec}$ for this condition, since velocity discrimination is very difficult for either the low or the high spatial (temporal) frequency stimulus at lower or higher velocities. As shown in Figure 4, the optimal discrimination thresholds for this condition are within the range of those obtained in the $1-1,0.25-0.25$, and 4-4 conditions (replotted in the same figure for comparison), and, at $2 \mathrm{deg} / \mathrm{sec}$, are about as precise as in the $1-1 \mathrm{con}$ dition. This result indicates that precise velocity discrimination remains possible for two stimuli whose spatial frequencies are separated by 4 octaves $(0.25-4 \mathrm{c} / \mathrm{deg})$. The absolute values of threshold for M.Q. at $2 \mathrm{deg} / \mathrm{sec}$ appears lower for the $0.25-4$ condition than for the 4-4 condition, whereas the thresholds for Y.C. for the two conditions overlap at this velocity because of the relatively large standard errors. M.Q.'s low thresholds may be related to the use of a low spatial frequency as the reference for the $0.25-4$ condition, which caused discrimination to be performed using a physical velocity of the $4 \mathrm{c} / \mathrm{deg}$ comparison grating lower than $2 \mathrm{deg} / \mathrm{sec}$, to equate it perceptually with that of the $0.25-\mathrm{c} / \mathrm{deg}$ grating (see below).

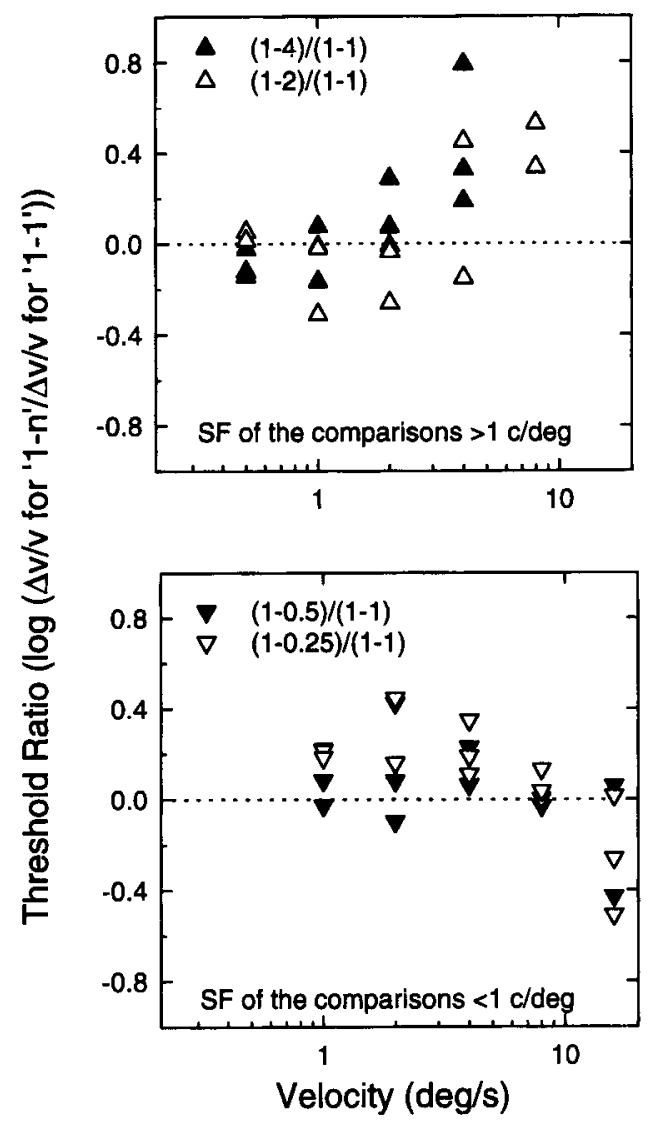

(a)

(b)

Figure 3. Log ratio of velocity discrimination thresholds for comparison stimuli with and without a spatial frequency difference. Panel (a) shows log $\mid(\Delta V / V$ for the $1-2$ or $1-4) /(\Delta V / V$ for 1-1)] as a function of velocity - that is, where the comparison stimulus is of a higher spatial frequency than the reference stimulus (1 c/deg). Panel (b) shows log $[(\Delta V / V$ for $1-0.25$ or $1-0.5) /$ $(\Delta V / V$ for 1-1)] as a function of velocity - that is, where the comparison stimulus is of a lower spatial frequency than the reference stimulus $(1 \mathrm{c} / \mathrm{deg})$. The letter $n$ on the $y$-axis symbolizes the spatial frequencies used for the comparison stimulus. 


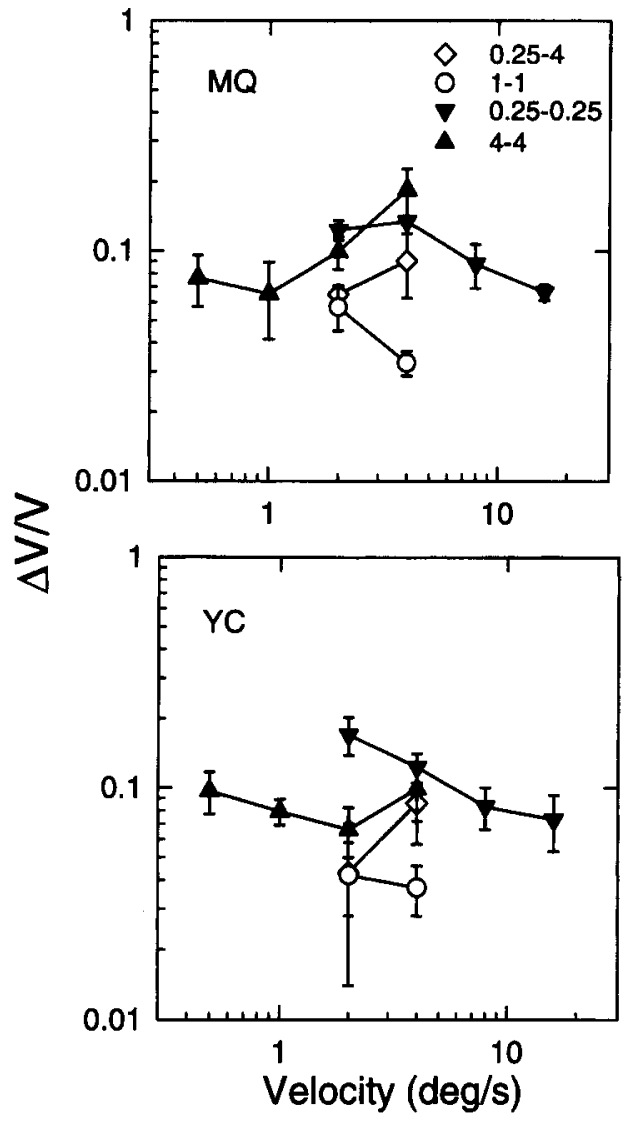

Figure 4. Velocity discrimination thresholds for $0.25-\mathrm{c} / \mathrm{deg}$ (reference) and 4-c/deg (comparison) gratings are shown as unfilled diamonds. Also plotted are threshold data obtained with 1-1, $0.25-0.25$, and $4-4$ grating pairs. The error bars show $\pm 1 S E M$. See Figure 2 for other details.

\section{Perceived Velocities}

In Figures 5 and 6, the effect of spatial frequency on perceived velocity is presented as velocity matching ratios, defined as log [reference velocity $(V) /$ comparison velocity $\left.\left(V_{\mathrm{B}}\right)\right]$. As expected, when both gratings are $1 \mathrm{c} / \mathrm{deg}$, velocity matching is accurate, as indicated by the data near the zero line. However, the data for the grating pairs of different spatial frequency show that, in order to be perceived as matching, (1) the physical velocities of the low spatial frequency comparison stimuli have to be greater than the velocity of the reference $(1 \mathrm{c} / \mathrm{deg})$, indicated by negative velocity matching ratios, and (2) the physical velocities of the high spatial frequency comparison stimuli have to be less than the velocity of the reference $(1 \mathrm{c} / \mathrm{deg})$, shown by positive velocity matching ratios. In other words, for two gratings moving at the same physical velocity, the high spatial frequency stimulus appears to move faster. The velocity matching ratios also vary modestly with velocity, as well as with spatial frequency. In general, the velocity matching ratio is closest to 1 at intermediate velocities, except for observer M.Q. for the 1-2 and 1-4 conditions (Figure 5). In Figure 6, the ve- locity matching ratios for 3 observers are pooled and plotted as a function of the spatial frequency of the comparison grating. The results show a monotonic increase of velocity matching ratio with spatial frequency. The fitted function indicates that perceived velocity varies with stimulus spatial frequency at a rate of about 0.19 $\log$ units of velocity per decade of spatial frequency.

\section{DISCUSSION}

\section{The Role of Temporal Frequency}

In order to code velocity, the motion energy provided by the visual stimulus must fall within the sensitive range of the spatiotemporal filters in the early stages of processing (Adelson \& Bergen, 1985). These filters' responses are

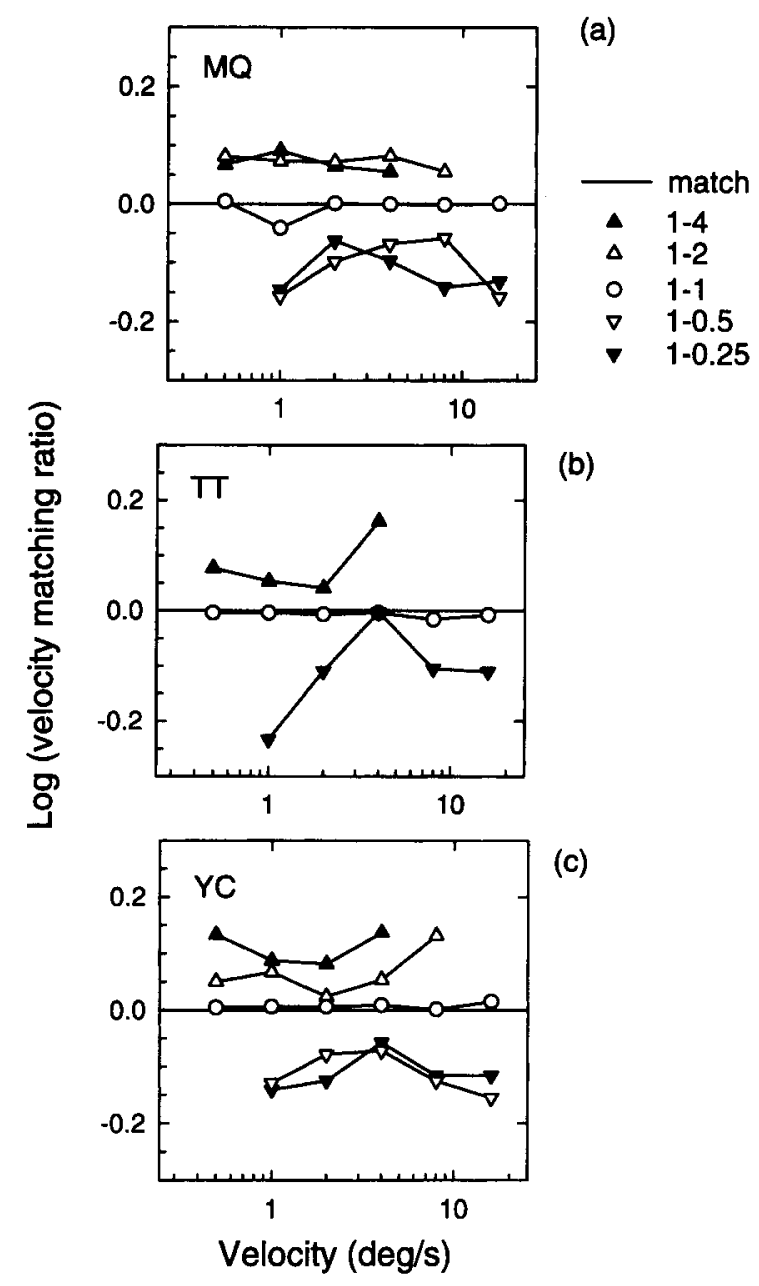

Figure 5. Biases in perceived velocity for different spatial frequencies. The velocity matching ratios for each spatial frequency, expressed as log $I($ reference velocity, $V) /($ comparison velocity, $\left.V_{B}\right)$, as a function of velocity. The dashed horizontal line at zero in each panel represents perfect velocity matching between the reference and the comparison stimuli. For clarity, the error bars are not presented; the average values of all measured standard errors are 0.012 for M.Q., 0.010 for T.T., and 0.010 for Y.C. See Figure 2 for other details. 


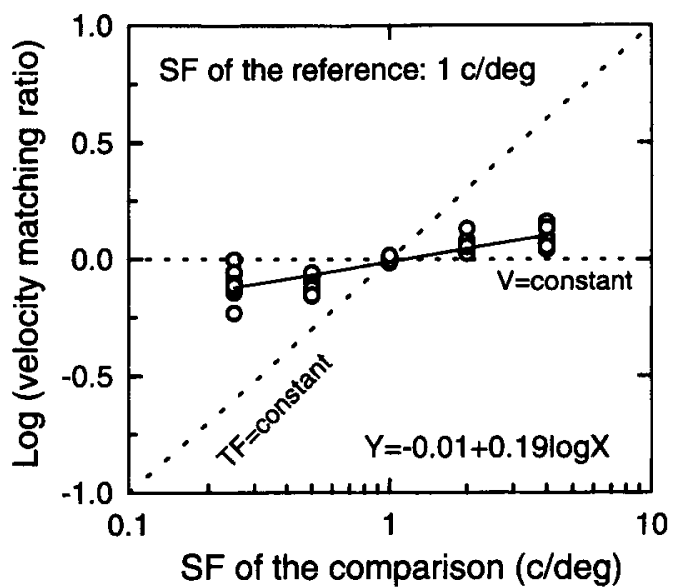

Figure 6. Velocity matching ratio as a function of spatial frequency. The dashed horizontal zero line represents perfect velocity matching between reference and comparison stimuli. Data for all velocities $-0.5,1,2,4,8$, and $16 \mathrm{deg} / \mathrm{sec}$-are pooled together. The solid line represents the best straight line fit to the data points.

either low pass or bandpass in both the spatial and the temporal domains (see, e.g., Kelly, 1979; Robson 1966), indicating that, above and below certain frequencies, a decreased amount of motion energy will be available for motion processing, and velocity discrimination could be impaired. As an extreme example, neither direction nor velocity information can be conveyed via these low-level filters when the temporal frequency of a moving stimulus exceeds the critical fusion frequency, which occurs for a given velocity when the spatial frequency is increased sufficiently. Conceivably, then, the precision of velocity discrimination might vary with the strength of the low-level filters' responses to suprathreshold moving stimuli. Figure 7 shows our velocity discrimination threshold data, obtained with grating pairs of $0.25,1$, and $4 \mathrm{c} / \mathrm{deg}$ (Figure 1), replotted as a function of temporal frequency. There is a general similarity for the three spatial frequencies to the temporal frequency tuning found previously in contrast detection experiments at a similar photopic mean luminance (see, e.g., Robson, 1966), with best performance at mid-range temporal frequencies. This similarity is consistent with the suggestion that differential sensitivity to temporal frequency could constrain the processing required for velocity discrimination, both when the reference and comparison gratings are of the same (Figures 1 and 7) and when they are of different spatial frequencies (Figure 2).

Previously, Thompson (1983) reported that optimal velocity discrimination for moving gratings occurs at a single mid-range temporal frequency, regardless of the stimulus spatial frequency. However, in his study, the contrast of each moving grating stimulus was adjusted to be an equal multiple of its detection threshold. This manipulation ensures that the variation of discrimination thresholds with temporal frequency (which was extremely large in Thompson's study) cannot be attributed to differences in the response strength of low-level spatiotemporal filters (see, also, Panish, 1988). Thompson attributed his finding to a crossover in the vicinity of $4 \mathrm{~Hz}$ between the sensitivities of two broadly tuned temporal channels, such as those proposed by Watson and Robson (1981), and interpreted his result as evidence that a comparison between the responses of these temporal channels underlies velocity coding. This interpretation, with which our data are also consistent, was subsequently elaborated in models of temporal frequency and velocity processing put forth by Smith and Edgar (1994) and Metha and Mullen (1997). As pointed out by Smith and Edgar (1994), a low-level velocity code based on comparing the responses in two broad temporal frequency channels does not preclude pooling across spatial frequency scales at a later stage in order to achieve explicit velocity tuning.

Our observers compared the velocities of gratings of different spatial frequency to determine whether the concomitant variation of temporal frequency would disrupt the precision of velocity discrimination. Like the results of previous spatial and temporal frequency randomization studies (McKee et al., 1986; Smith, 1987; Smith \& Edgar, 1991), our data indicate that the discrimination of low velocities remains precise when observers compare moving gratings of different spatial frequencies. However, unlike Smith (1987; Smith \& Edgar, 1991), we found no decrease in the precision of discrimination for velocities of 8 and $16 \mathrm{deg} / \mathrm{sec}$ when the reference and comparison gratings differed in spatial frequency. Smith and Edgar acknowledged that their results were consistent with the presence of mechanisms that encode stimulus velocity explicitly, but surmised that their observers'

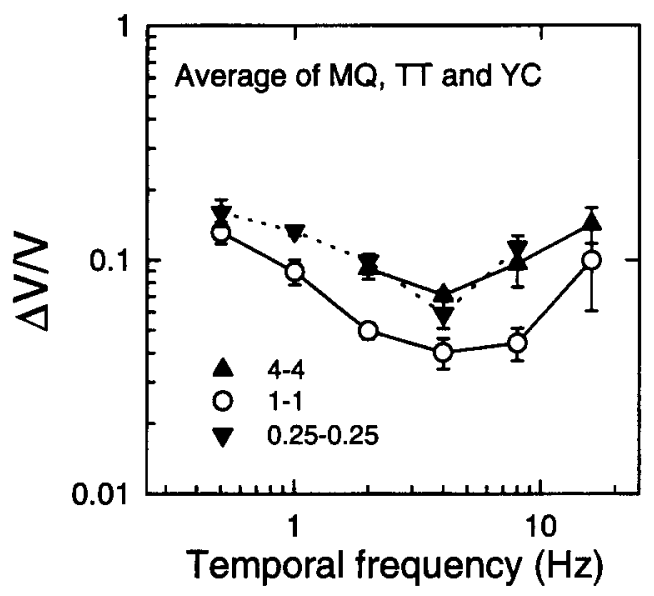

Figure 7. Velocity discrimination thresholds as a function of temporal frequency. The temporal frequencies, plotted on the $x$ axis, are converted from the velocities and spatial frequencies $(0.25,1$, or $4 \mathrm{c} / \mathrm{deg})$ of the individual stimuli. The data points represent the average for 3 observers. Note that the lowest temporal frequency for which data at $4 \mathrm{c} / \mathrm{deg}$ appears is $2 \mathrm{~Hz}$, which corresponds to $0.5 \mathrm{deg} / \mathrm{sec}$, and the highest temporal frequency for $0.25 \mathrm{c} / \mathrm{deg}$ is $8 \mathrm{~Hz}$, which corresponds to $32 \mathrm{deg} / \mathrm{sec}$. 
judgments were influenced simultaneously by information about stimulus temporal frequency. Clearly, if observers based velocity discriminations on both the velocity and the temporal frequency of the moving stimuli, impaired performance would be expected when the spatial frequencies of the gratings vary within a trial. In our study, the observers may have been discouraged from using temporal frequency information because the spatial frequencies of the reference and comparison stimuli varied by at least one octave, compared with only $\pm 12.5 \%$ in the experiments by Smith and by Smith and Edgar (1991). The resulting temporal frequency differences between the stimuli in our study may have been so large that the observers could readily discount them as a basis for discrimination. Another difference between our study and those of Smith and Smith and Edgar (1991) is that we presented the moving gratings for $200 \mathrm{msec}$, as compared with 1 or $2 \mathrm{sec}$ in their experiments. Nevertheless, velocity discrimination in our study was precise, which is consistent with the report by McKee and Welch (1985) that optimal velocity discrimination for line targets requires an exposure duration of only $100 \mathrm{msec}$ or less.

\section{Perceived Velocity}

In addition to discrimination thresholds, the present study also provides data on perceived velocity as a function of spatial frequency. Previously published data on this issue yielded conflicting results. Some studies reported that moving stimuli of high spatial frequencies are perceived to be faster (Ferrera \& Wilson, 1991; McKee et al., 1986), consistent with our results (see Figures 5 and 6). Another study reported the opposite-namely, that for two gratings moving at the same physical velocity, the one of higher spatial frequency appeared to move more slowly (Smith \& Edgar, 1990). Recently, Yo and Wilson (1993) reported that, in the peripheral visual field, stimulus temporal frequency and velocity generally were considerably underestimated, but discrimination along both dimensions was precise. Possibly, judgments of perceived magnitude and discrimination do not follow the same coding principle or do not occur at the same stage of processing.

\section{Velocity Coding and the Combination of Signals in the Spatial Domain}

The combination of motion signals in the spatial frequency domain has been of major concern in some recent studies that measured velocity discrimination. For example, two studies using random-dot cinematograms found that velocity discrimination thresholds were unaffected by manipulations that altered the spatial frequency content of the stimulus (Smith, Snowden, \& Milne, 1994; Watamaniuk, Grzywacz, \& Yuille, 1993), and, in one of these studies, as in ours, discrimination remained precise despite changes in perceived velocity (Watamaniuk et al., 1993). Recently Yang and Blake (1994) reported that sensitivity to a coherent-motion stimulus with limited spatial frequency content can be affected by a noise mask con- sisting of other spatial frequency components, suggesting that the underlying motion-sensitive mechanisms are not spatial frequency specific. Although our present study does not directly address how velocity information is combined or compared across spatial frequency, we have addressed this issue in two companion studies. In one, we found that adaptation to moving gratings of one spatial frequency $(1 \mathrm{c} / \mathrm{deg})$ affected velocity discrimination at other spatial frequencies $(0.25 \mathrm{c} / \mathrm{deg}$ and $4 \mathrm{c} / \mathrm{deg})$, indicating that the mechanisms underlying velocity discrimination are not narrowly tuned to spatial frequency (Chen, Bedell, \& Frishman, 1996). In the other, we found that uncertainty, introduced by varying the spatial frequency of the reference and comparison stimuli presented from trial to trial, does not elevate velocity discrimination thresholds, as would be expected if the mechanisms underlying velocity discrimination were tuned to spatial frequency (Chen, Bedell, Frishman, \& Levi, 1998). On the other hand, discrimination thresholds are impaired when the velocity of the reference stimulus varies from trial to trial, consistent with the operation of velocity-tuned mechanisms in velocity discriminations. Supporting (albeit limited) neurophysiological evidence comes from preliminary reports that some neurons in cortical area MT are very broadly tuned to spatial frequency but nevertheless maintain velocity tuning (Movshon, Newsome, Gizzi, \& Levitt, 1988; Newsome et al., 1983).

Finally, it should be noted that the insusceptibility of precise discrimination to changes in a nondiscriminated dimension is not unique to velocity coding. For example, discrimination of line orientation and of spatial interval is not affected by the spatial location of the comparison stimuli (Paradiso, Carney, \& Freeman, 1989), and discrimination of orientation is independent of variations in spatial frequency between the reference and the comparison stimuli (Heeley, Buchanan-Smith, \& Heywood, 1993 ). Within the domain of velocity perception, alternating the directions of movement between comparison stimuli has been shown to have little effect on the precision of velocity discrimination (Chen, 1994; Smith \& Edgar, 1991). These instances of precise discrimination between stimuli that presumably activate separate groups of neurons at the early stages of visual processing suggest that discrimination along specific dimensions, such as velocity, may be made at higher processing stages, after information is integrated across the nondiscriminated dimensions.

\section{REFERENCES}

Adelson, E. H., \& Bergen, J. R. (1985). Spatiotemporal energy models for the perception of motion. Journal of the Optical Society of America $A, 2,284-299$.

Blakemore, C., \& Campbell, F. W. (1969). On the existence of neurones in the human visual system selectively sensitive to the orientation and size of retinal images. Journal of Physiology, 203, 237-260.

Chen, Y. (1994). Speed discrimination upon stimuli moving in the same or opposite directions [Abstract]. Investigative Ophthalmology \& $V_{i}$ sual Science, 35 (Suppl.), 1268.

Chen, Y., Bedell, H. E., \& Frishman, L. J. (1996). The effects of 
cross-spatial-frequency adaptation on speed discrimination. In Vision science and its applications (Technical Digest Series, Vol. 4, pp. 140143). Washington, DC: Optical Society of America.

Chen, Y., Bedell, H. E., Frishman, L. J., \& Levi, D. M. (1998). Stimulus uncertainty affects velocity discrimination. Vision Research, $\mathbf{3 8}$, 1265-1272.

DeValois, R. L., Albrecht, D. G., \& Thorell, L. G. (1982). Spatial frequency selectivity of cells in macaque visual cortex. Vision Research, 22, 545-559.

Ferrera, V. P., \& Wilson, H. R. (1991). Perceived velocity of moving two-dimensional patterns. Vision Research, 31, 877-893.

Grzywacz, N. M., \& Yuille, A. L. (1990). A model for the estimate of local image velocity by cells in the visual cortex. Proceedings of the Royal Society of London: Series B, 239, 129-161.

HEEGER, D. J. (1987). Model for extraction of image flow. Journal of the Optical Society of America, 4, 1455-1471.

Heely, D. W., Buchanan-Smith, H. M., \& Heywood, S. (1993). Orientation acuity for sine-wave gratings with random variation of spatial frequency. Vision Research, 33, 2509-2513.

KELLY, D. H. (1979). Motion and vision. II: Stabilized spatio-temporal threshold surface. Journal of the Optical Society of America, 69, 1340-1349.

Maunsell, J. H. R., \& VAN Essen, D. C. (1983). Functional properties of neurons in middle temporal visual area of the macaque monkey. I: Selectivity for stimulus direction, velocity, and orientation. Journal of Neurophysiology, 49, 1127-1147.

McKee, S. P., Silverman, G. H., \& Nakayama, K. (1986). Precise velocity discrimination despite random variations in temporal frequency and contrast. Vision Research, 26, 609-619.

MCKEE, S. P., \& WELCH, L. (1985). Sequential recruitment in the discrimination of velocity. Journal of the Optical Society of America A, 2, 243-251.

Metha, A. B., \& Mullen, K. T. (1997). Red-green and achromatic temporal filters: A ratio model predicts contrast-dependent speed perception. Journal of the Optical Society of America A, 14, 984-996.

Movshon, J. A., \& Newsome, W. T. (1996). Visual response properties of striate cortical neurons projecting to area MT in macaque monkeys. Journal of Neuroscience, 16, 7733-7741.

Movshon, J. A., Newsome, W. T., Gizzi, M. S., \& LevitT, J. B. (1988). Spatio-temporal tuning and velocity sensitivity in macaque visual cortical neurons [Abstract]. Investigative Ophthalmology \& Visual Science, 25 (Suppl.), 327.

Movshon, J. A., Thompson, P., \& Tolhurst, D. J. (1978). Spatial and temporal contrast sensitivity in area $17 \& 18$ of cat's cortex. Vision Research, 18, 101-120.

Newsome, W. T., Gizzı, M. S., \& Movshon, J. A. (1983). Spatial and temporal properties of neurones in macaque MT [Abstract]. Investigative Ophthalmology \& Visual Science, 24 (Suppl.), 106.

Orban, G. A., DE WOLF, J., \& MAEs, H. (1984). Factors influencing velocity coding in the human visual system. Vision Research, 23, 33-39.
Panish, S. C. (1988). Velocity discrimination at constant multiples of threshold contrast. Vision Research, 28, 193-201.

Paradiso, M. A., Carney, T., \& Freeman, R. D. (1989). Cortical processing of hyperacuity tasks. Vision Research, 29, 247-254.

Robson, J. G. (1966). Spatial and temporal contrast sensitivities functions of visual system. Journal of the Optical Society of America, 56, 1141-1142.

SMITH, A. T. (1987). Velocity perception and discrimination: Relation of temporal mechanisms. Vision Research, 27, 1491-1500.

Smith, A. T., \& Edgar, G. K. (1990). The influence of spatial frequency on perceived temporal frequency and perceived speed. Vision Research, 30, 1467-1474.

Sмiтh, A. T., \& Edgar, G. K. (1991). The separability of temporal frequency and velocity. Vision Research, 31, 321-326.

SmITH, A. T., \& EDGAR, G. K. (1994). Antagonistic comparison of temporal filter outputs as a basis for velocity perception. Vision Research, 34, 253-265.

Smith, A. T., SNOWDEN, R. J., \& MiLne, A. B. (1994). Is global motion really based on spatial integration of local motion signals? Vision Research, 34, 2425-2430.

Thompson, P. (1983). Discrimination of moving gratings at and above detection threshold. Vision Research, 23, 1533-1538.

Watamaniuk, S. N. J., GrzYwacz, N. M., \& Yuille, A. L. (1993). Dependence of velocity and direction perception on cinematogram dot density. Vision Research, 33, 849-859.

WATSON, A. B., \& RoBson, J. G. (1981). Discrimination at threshold: Labelled detectors in human vision. Vision Research, 21, 1115-1122.

Wilson, H. R., MCFarlane, D. K., \& Philip, G. C. (1984). Spatial frequency tuning of orientation selective units estimated by oblique masking. Vision Research, 23, 873-882.

YANG, Y., \& BLAKE, R. (1994). Broad tuning for spatial frequency of neural mechanisms underlying visual perception of coherent motion. Nature, 324, 369-372.

Yo, C., \& Wilson, H. R. (1993). Peripheral temporal frequency channels code frequency and speed inaccurately but allow accurate discrimination. Vision Research, 33, 33-45.

\section{NOTE}

1. Because both stimuli always moved in the same direction, this study more precisely studied speed discrimination rather than velocity discrimination. However, previous studies showed that discrimination is equally good whether two gratings of the same spatial frequency move in the same direction or in opposite directions (Chen, 1994; Smith \& Edgar, 1991). We will, therefore, use the term velocity discrimination, which is more inclusive.

(Manuscript received June 27, 1997; revision accepted for publication November $18,1997$. 